Document downloaded from:

http://hdl.handle.net/10251/45126

This paper must be cited as:

Pérez Soler, J.; Llorente Sáez, R. (2014). Hybrid UWB and WiMAX radio-over-multi-mode fibre for in-building optical networks. Journal of Optics. 16(1):1-6. doi:10.1088/20408978/16/1/015401.

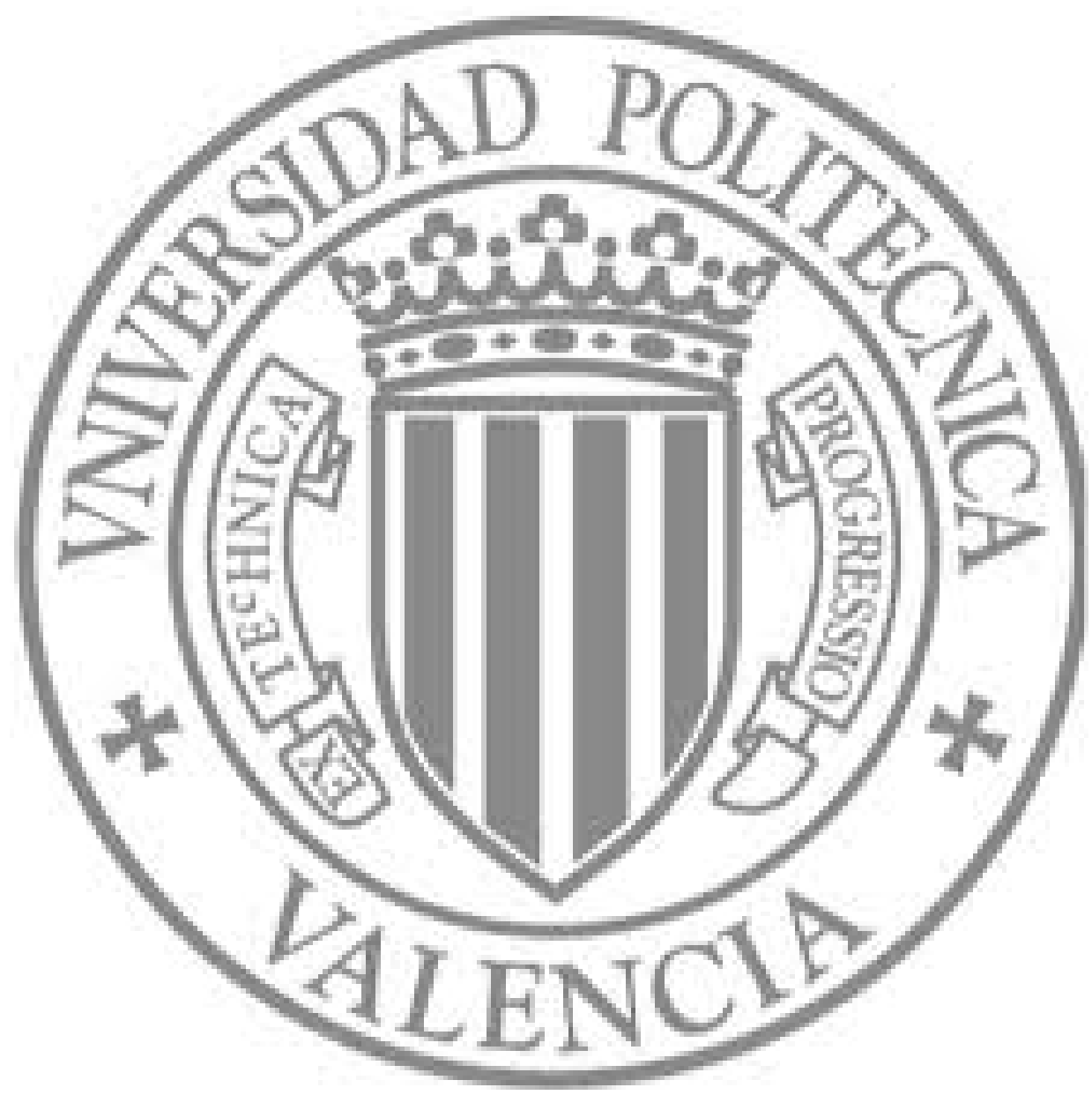

The final publication is available at

http://dx.doi.org/10.1088/2040-8978/16/1/015401

Copyright

IOP Publishing: Hybrid Open Access 


\title{
Hybrid UWB and WiMAX Radio-over-Multi-Mode Fibre for In-Building Optical Networks
}

\author{
J Perez ${ }^{1,3}$ and R Llorente ${ }^{2}$ \\ ${ }^{1}$ Optical Communications Research Group, Northumbria Communications Research Lab, \\ Faculty of Engineering and Environment, Northumbria University, Newcastle-upon-Tyne, NE1 \\ 8ST, UK \\ ${ }^{2}$ Nanophotonics Technology Center, Universitat Politècnica de València, Camino de Vera, \\ 46022 Valencia, Spain, \\ E-mail: joaquin.perez@northumbria.ac.uk
}

\begin{abstract}
In this letter the use of hybrid WiMedia-defined Ultra-Wideband (UWB) and IEEE 802.16d WiMAX radio-over-fibre is proposed and experimentally demonstrated for multimode based in-building optical networks with the advantage of great immunity to optical transmission impairments. In the proposed approach, spectral coexistence of both signals must be achieved with negligible mutual interference. The experimental study performed addressed an indoor configuration with $50 \mu \mathrm{m}$ multi-mode fibres (MMF) and $850 \mathrm{~nm}$ Vertical-Cavity Surface-Emitting Lasers (VCSEL) transmitters. The results indicate that the impact of the wireless convergence in radio-over-multi-mode fibre (RoMMF) is significant for UWB transmissions mainly due to MMF dispersion and electrooptical (EO) devices with limited bandwidth. On the other hand, a WiMAX transmission is feasible for a $300 \mathrm{~m} \mathrm{MMF}$ and $30 \mathrm{~m}$ wireless link in presence of UWB, with $-31 \mathrm{dBm}$ WiMAX EVM.
\end{abstract}

Keywords: Ultra-wideband, WiMAX, radio-over-fibre, multi-mode fibre, convergence

\section{Introduction}

UWB is a radio communications technology targeting a high bit rate short-range indoor link with low power consumption, high tolerance to multipath fading and low deployment cost [1]. UWB is an unlicensed wireless service allocated within the $3.1-10.6 \mathrm{GHz}$ band in current regulation [2-5]. Different UWB signal formats implementations exist mainly based on impulse radio and orthogonal frequency division multiplexing (OFDM) [5]. However, the UWB WiMedia-defined implementations based on multi-band OFDM (MB-OFDM) modulation [6] are deeply commercially exploited in short-range environments, e.g. computer peripheral interconnection, namely wireless universal serial bus (wireless-USB) [7], and in high-definition audio/video streaming from the computer to the TV set. Due to the unlicensed nature of UWB spectrum, a spectral overlapping is present with the spectrum of already existing narrowband and wideband systems, such as WiMAX $802.16 \mathrm{~d} / \mathrm{e}$ (3.3 to $3.8 \mathrm{GHz}$ and 5.4 to $5.825 \mathrm{GHz}$, respectively) or IEEE 802.11 a devices (in the band from 4.9 to $5.85 \mathrm{GHz}$ ). WiMAX is a wireless transmission technology targeting a medium- to long- range data communications [8]. On the other hand, WiMAX applications are addressed to improve the coverage in large wireless local

${ }^{3}$ This work was carried out at Nanophotonics Technology Center, Universitat Politècnica de València, Camino de Vera, 46022 Valencia, Spain 
area network (WLAN) installations in special scenarios such as high speed trains communications, indoor broadcasting or in punctual massive sports events coverage [9-11]. Regarding these features WiMAX and UWB are expected to coexist on indoor scenarios.

In-building optical networks provide coexistence scenarios for UWB and WIMAX end-user applications. MMF is predominantly used worldwide over single-mode fibre (SMF) for indoor links up to $300 \mathrm{~m}$, and offers easier installation in within-building environments compared to SMF due the large core diameter of MMF fibres (typically 50 or $62.5 \mu \mathrm{m}$ ) [12]. With the interest in low-cost radio-over-fibre (RoF) solutions, indoor installations are typically based on MMF, VCSELs operating at $850 \mathrm{~nm}$ and low-cost photoreceivers [13]. The distribution of WiMAX and UWB-over-fibre has been studied previously for long-reach passive optical networks (PON) [14, 15]. The target of WiMAX RoF applications is to improve the coverage of broadband services in distributed antenna systems (DAS) applications [16], and UWB RoF applications are required to overcome range limitations and develop cellular capabilities on UWB networks [17-19].

Moreover; the impact of harmful interference between UWB and WiMAX in the $3.1-4.2 \mathrm{GHz}$ band was studied in [20] within an analytical framework. A first approach of this analysis has been presented by the authors at [21]. However, the most of the publications had analysed each wireless communication system impact separately $[10,22,23]$. Therefore, in this paper the impact of interference in MB-OFDM UWB over WiMAX 802.16d on a hybrid RoMMF architecture and a final radio link to the end-user system is presented.

This paper is organized as follows; Section 2 describes the experimental optical wireless convergence setup. In Section 3 the results of RoMMF convergence transmission of WiMAX and MB-OFDM UWB are discussed, and considering the radio link path are included in Section 4. Finally, conclusions are drawn.

\section{Experimental Setup}

The experimental setup in figure 1 is implemented in order to evaluate the performance of MB-OFDM UWB and WiMAX 802.16d joint distribution architecture in a RoMMF system with a radio link path to a final user. The setup is divided in three sections, head-end, fibre transmission and radio link to end-user.

Table 1. WiMAX 802.16d and WiMedia-defined UWB signals under study parameters

\begin{tabular}{ll}
\hline \multicolumn{2}{c}{ WiMAX 802.16d parameters } \\
\hline Time-frequency coding & TFC1 \\
Band frequency & Band group \#1 \\
Centre frequency & $33.168-4.752 \mathrm{GHz}\}$ \\
Bit rate, Modulation & $200 \mathrm{GHz}$ \\
EIRP & $-41.3 \mathrm{dBm} / \mathrm{MHz}$ \\
\hline \multicolumn{2}{c}{ MB-OFDM UWB parameters } \\
\hline Centre frequency & $3.5 \mathrm{GHz}$ \\
Bandwidth & $3.5 \mathrm{MHz}$ \\
FFT-points & 256 \\
Subchannel spacing & $15.625 \mathrm{kHz}$ \\
Data Rate & $13.1 \mathrm{Mbit} / \mathrm{s}$ \\
Modulation & $64 \mathrm{QAM}$ \\
\hline
\end{tabular}

In the first section, the head-end, MB-OFDM UWB and IEEE.802.16d WiMAX signals are generated, then converted from the electrical to the optical domain by the VCSEL and launched into the MMF. The WiMAX signal has been generated according to the IEEE 802.16d standard [8]. The UWB signal has been generated fulfilling the WiMedia-defined UWB specification [3] by an evaluation kit transceiver. The UWB signals perform bitrate of $200 \mathrm{Mbit} / \mathrm{s}$ over a $500 \mathrm{MHz}$ bandwidth 
and the WiMAX signals perform a bit rate of $13.1 \mathrm{Mbit} / \mathrm{s}$ over $3.5 \mathrm{MHz}$. Main WiMAX and UWB signal parameters are summarized in Table 1. A low-noise amplifier (LNA) and RF attenuation pair has been used after UWB generation previous VCSEL to maximize EO conversion. The VCSEL used on this setup is designed for $850 \mathrm{~nm}$ up to $4.25 \mathrm{Gbit} / \mathrm{s}$.

The optical signal is transmitted over 100, 200, 300 and $400 \mathrm{~m}$ links of $50 \mu \mathrm{m}$ core MMF; each with $500 \mathrm{MHz} \cdot \mathrm{km}$ modal bandwidth. An optical attenuator is used before the MMF in order to prevent receiver distortion and test purposes. After the fibre transmission, the optical signal is converted back to an electrical signal using the $850 \mathrm{~nm}$ PIN-TIA photoreceiver. Then, after the LNA, with $\mathrm{G}=40 \mathrm{~dB}$ and $\mathrm{NF}=2.5 \mathrm{~dB}$, the RF signal is delivered to a $7.5 \mathrm{dBi}$ antenna designed for indoor applications. The final wireless transmission section considers simultaneous radio transmission of UWB and WiMAX signals over different link lengths. In the reception user-end side each signal is received with a MB-OFDM UWB omni $2 \mathrm{dBi}$ and WIMAX subscriber directional $9 \mathrm{dBi}$ antennas, respectively. Then both signals are captured and processed to measure quality factors, e.g., error vector magnitude (EVM). The EVM is considered a figure of merit for assessing the quality of digitally modulated communication signals of each received constellation.

(a)
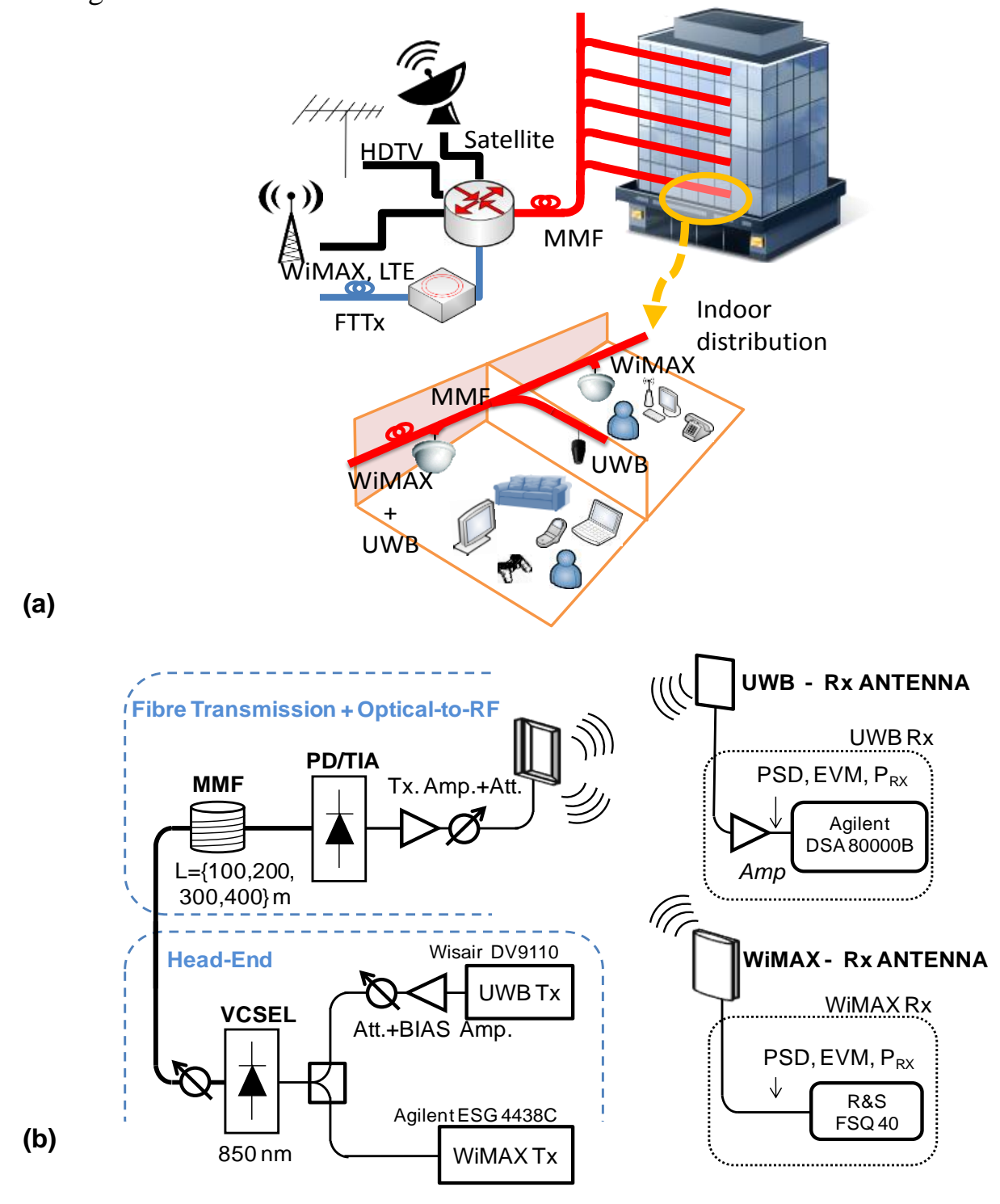

Figure 1. (a) Application scenario and (b) experimental setup to study the performance of the optical wireless hybrid architecture. 


\section{Multi-mode fibre transmission}

In a first approach the transmission of MB-OFDM UWB and WiMAX 802.16d signals independently over MMF is evaluated. In the figure 2 the measured EVM after photodiode for each MMF link length and the back-to-back case is depicted for each signal. In the figure 2(a) it can be observed that the EVM remains under the $-14.5 \mathrm{~dB}$ EVM threshold for successful UWB communication [3], in almost all configurations. In figure 2(b), the EVM results indicate that a low RF power is the main cause of degradation, since EVM measurements remain under a -31 dB EVM threshold for a 64 QAM 802.16d WiMAX communication [8]. In both cases, MMF modal dispersion for each link length is reflected as a linear degradation of EVM results. For example, EVM values for a $400 \mathrm{~m}$ MMF link experienced an intense degradation of both signals at any RF power launched. According to these EVM results, it is possible to find a combination of UWB and WiMAX RF power values where MMF impairments are the main cause of EVM degradation instead of the noise introduced by the RF power launched, e.g., 0 to $3 \mathrm{dBm}$ for WiMAX and -6 to $-3 \mathrm{dBm}$ for UWB in-band RF power. In order to ensure RF coexistence, the amplifier-attenuator pair at the head-end section is adjusted to meet a $35 \mathrm{~dB}$ ratio between WiMAX and UWB, which is required for a correct 64 QAM demodulation [8].

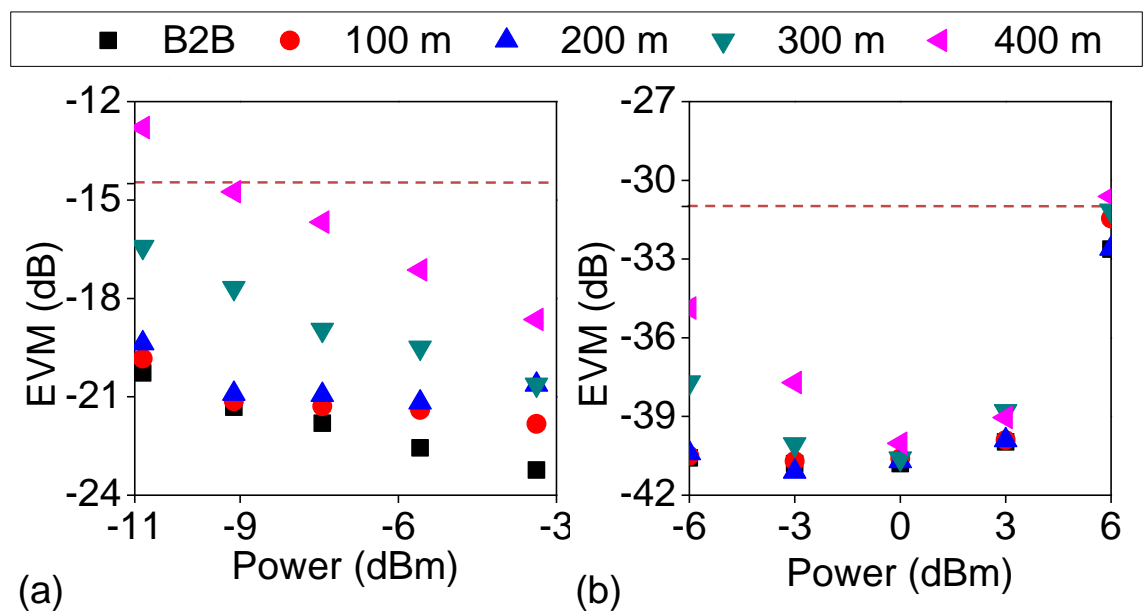

Figure 2. Measured EVM after MMF transmission for (a) MB-OFDM UWB and (b) WiMAX 802.16d signal as a function of different RF power launched at the VCSEL.

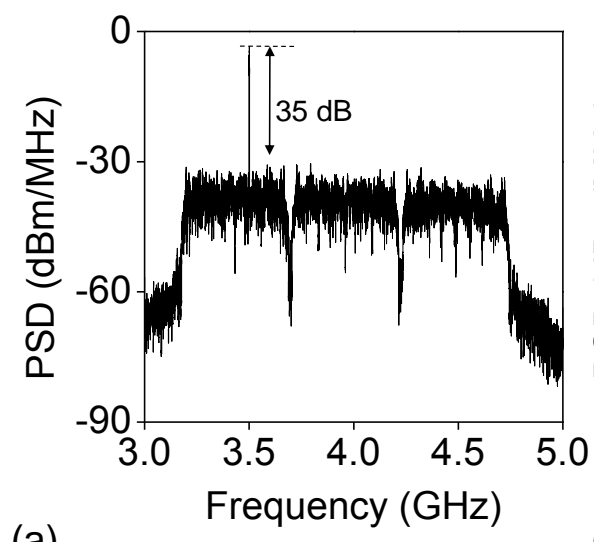

(a)

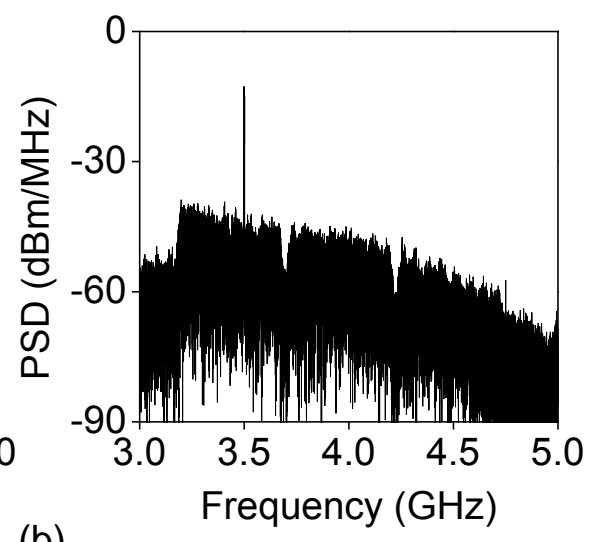

(b)

Figure 3. UWB and WiMAX PSD spectrum measured (a) before VCSEL and (b) after $400 \mathrm{~m}$ MMF simultaneous transmission, $\mathrm{RBW}=1 \mathrm{MHz}$. 
The simultaneous RF spectrum for a $3 \mathrm{dBm}$ WiMAX and $-6 \mathrm{dBm}$ UWB band power signals before the VCSEL modulation is represented at figure 3(a). This head-end RF power configuration accomplished with the relative $35 \mathrm{~dB}$ margin. Figure $3(\mathrm{~b})$ shows the received spectrum after a transmission over $400 \mathrm{~m}$ MMF. In this experimental set-up the spectral mask power, according to the regulations, is adjusted before wireless transmission. These figures indicate that the filtering effect due to modal dispersion and to limited bandwidth components compared with UWB signal bandwidth is significant. These effects introduce important degradation on the signal after MMF transmission, as studied in [18].
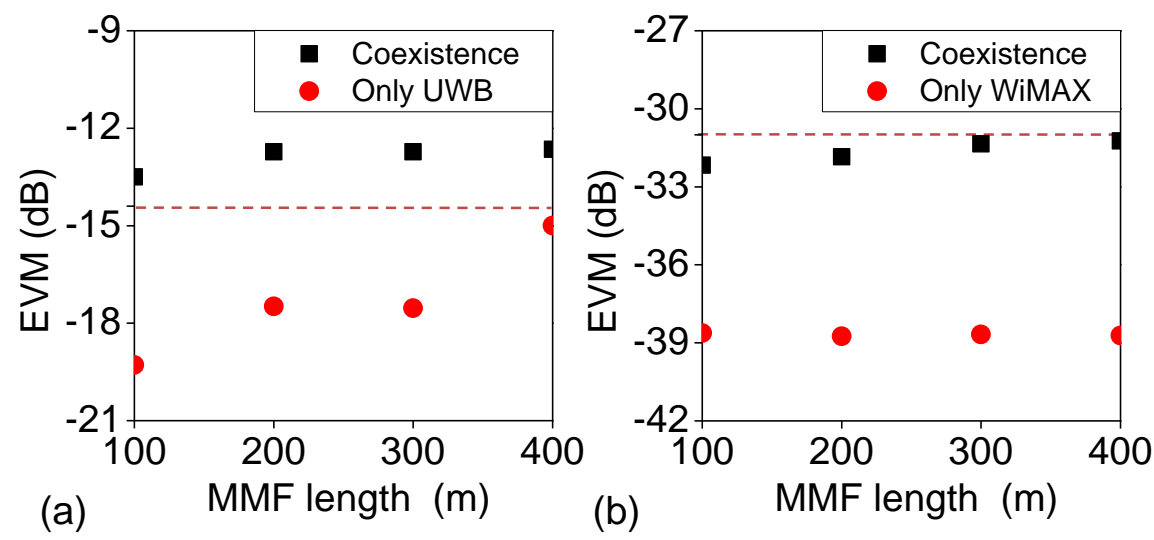

Figure 4. EVM measurements for (a) MB-OFDM and (b) 802.16d WiMAX in coexistence and individual transmission over different MMF links.

In order to study the impact of MB-OFDM UWB over WiMAX 802.16d in simultaneous MMF transmission, the EVM has been measured according to the previous RF power head-end adjustments. The EVM measured for both signals over each MMF link are represented in figure 4. The EVM UWB degradation due to WiMAX simultaneous transmission over MMF is depicted in figure 4(a). The threshold for a successful UWB communication of $-14.8 \mathrm{~dB}$ EVM is depicted as a dashed line. The UWB EVM at $100 \mathrm{~m}$ MMF increases from $-19.1 \mathrm{~dB}$ to $-13.5 \mathrm{~dB}$ compared with isolated UWB MMF transmission. In figure 4(b) the EVM WiMAX is represented and the $-31 \mathrm{~dB}$ EVM WiMAX threshold as a dashed line. The EVM WiMAX degradation at $100 \mathrm{~m}$ MMF link due to simultaneous distribution increases from $-38.6 \mathrm{~dB}$ to $-32.1 \mathrm{~dB}$. The EVM degradation indicates that WiMAX is more robust in RoMMF simultaneous distribution than UWB, due to MMF modal dispersion and EO components with limited bandwidth effects.

\section{Radio-over-fibre transmission}

The performance of the complete system considering the wireless transmission section is evaluated. The EVM results for each received constellation link are analysed for each case; on a UWB isolated transmission and over a coexistence RoMMF link for each dedicated UWB and WiMAX user-end receiver. The complete RoMMF system is evaluated over MMF links from $100 \mathrm{~m}$ to $400 \mathrm{~m}$, a UWB wireless link of $\{1,2,3\} \mathrm{m}$ and a WiMAX wireless link of $\{12,20,30\} \mathrm{m}$. 


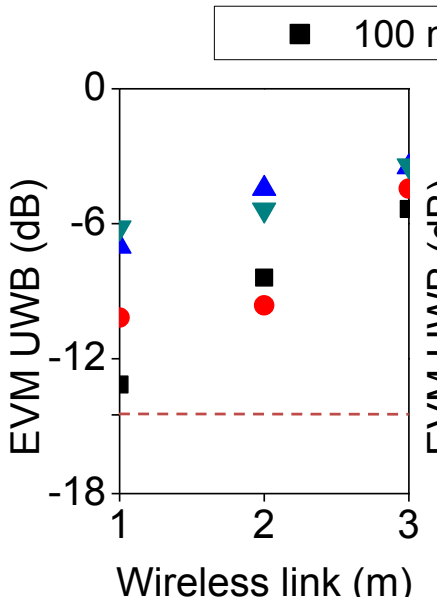

(a)

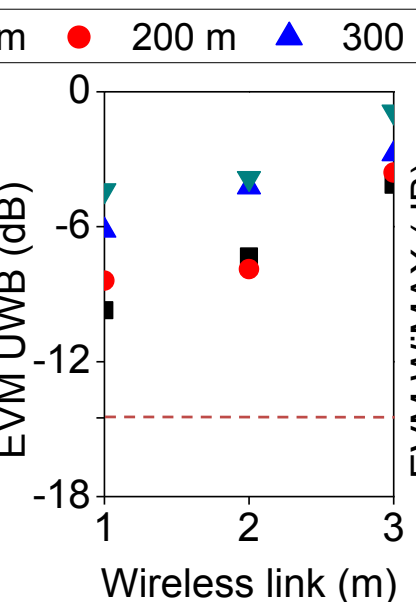

(b)

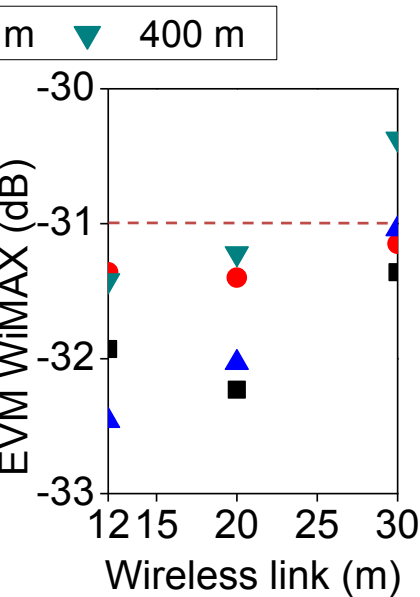

(c)

Figure 5. EVM measurements for (a) isolated UWB RoMMF link, simultaneous distribution evaluating (b) UWB RoMMF link and (c) WiMAX RoMMF link.

The impact of simultaneous UWB and WIMAX RoMMF distribution over each wireless communications end-user is represented in figure 5. In the case of the WIMAX impact over UWB a clear degradation is observed in figure 5(b) compared with the isolated case in figure 5(a). However, this impact in the wireless link is not as significative as the MMF transmission impairments explained in the previous section. For example, from -9.62 dB EVM to -7.88 dB EVM for $200 \mathrm{~m}$ MMF link over a $2 \mathrm{~m}$ wireless link for UWB receiver due to simultaneous transmission.

In the other case, the impact of UWB over WiMAX at wireless receiver-end still enables a successful communication up to $30 \mathrm{~m}$ wireless range for $300 \mathrm{~m}$ MMF link and $20 \mathrm{~m}$ for $400 \mathrm{~m}$ MMF link, as depicted in figure 5(c). These results indicate that the main impairments in the simultaneous RoMMF distribution are mostly located in the MMF transmission section rather than in the radio link path to the end-user.

\section{Conclusion}

The experimental results of this new hybrid MB-OFDM UWB and WiMAX 802.16d simultaneous distribution over a RoMMF architecture indicate the feasibility of hybrid high bit rate over a short range fibre link distance, below $100 \mathrm{~m}$ MMF link, and a medium bit rate, only WiMAX, for a long range distance up to $300 \mathrm{~m}$ MMF link. In the WIMAX case the results indicates that the main impairments are located at MMF transmission section, where the UWB presence degrade about $6 \mathrm{~dB}$ WiMAX EVM the transmission for each link. However, in the wireless section the impact of MB-OFDM UWB allows a 30m WIMAX wireless link from a 300m MMF path. In the case of the UWB communication due to the MMF modal dispersion the UWB suffers an important degradation. Furthermore, the impact of WiMAX over UWB implies degradation on EVM terms in all the RoMMF system limiting the quality of the UWB communications. The evaluation of EVM results indicates that in this setup the WiMAX communication is feasible under simultaneous distribution with UWB over RoMMF systems, e.g., up to $30 \mathrm{~m}$ WiMAX wireless link for a $300 \mathrm{~m}$ MMF link transmission. Moreover, the wireless convergence RoMMF performance should be improved with more linear and wider bandwidth systems components, e.g., VCSEL and photoreceivers, accordingly to the total RF bandwidth objective in the system.

\section{Acknowledgements}

Support from Spanish National Plan project MODAL TEC2012-38558-C02-01 is acknowledged 


\section{References}

[1] Kohno R 2004 State of arts in ultra wideband (UWB) wireless technology and global harmonization. In: Microwave Conference, 2004. 34th European, (New York: IEEE) pp 1093-9

[2] FCC 2002 Revision of Part 15 of the Commission's Rules regarding ultra wideband transmission systems. (Washington: Federal Communications Commission (FCC))

[3] ECMA 2008 High rate ultra wideband PHY and MAC Standard (Geneva: ECMA)

[4] ECC $2006 \mathrm{ECC} / \mathrm{DEC} /(06) 04$ : On the harmonised conditions for devices using ultra wideband (UWB) technology in bands below $10.6 \mathrm{GHz}$. (Copenhagen: Electronic Communications Committee - ECC)

[5] Llorente R, Alves T, Morant M, Beltran M, Perez J, Cartaxo A and Marti J 2008 Ultra-wideband radio signals distribution in FTTH networks IEEE Photonics Technology Letters 20 945-7

[6] Pavon J P, Sai Shankar N, Gaddam V, Challapali K and Chun-Ting C 2006 The MBOA-WiMedia specification for ultra wideband distributed networks Communications Magazine, IEEE 44 128-34

[7] Heidari G 2008 WiMedia UWB: technology of choice for wireless USB and Bluetooth (New York: Wiley)

[8] IEEE 2004 IEEE Standard for Local and Metropolitan Area Networks Part 16: Air Interface for Fixed Broadband Wireless Access Systems. In: IEEE Std 802.16-2004 (Revision of IEEE Std 802.16-2001), (New York, NY, USA: IEEE) pp 1-857

[9] Katz M D and Fitzek F H P 2009 WiMAX evolution: emerging technologies and applications (New York: Wiley)

[10] Chien-Hung Y, Chi-Wai C, Yen-Liang L, Sz-Kai W, Shi-Yang C, Chorng-Ren S, Min-Chien T, Jiunn-Liang L, Dar-Zu H and Sien C 2010 Theory and Technology for Standard WiMAX Over Fiber in High Speed Train Systems Journal of Lightwave Technology 28 2327-36

[11] Chowdhury A, Hung-Chang C, Yu-Ting H and Gee-Kung C 2009 Advanced System Technologies and Field Demonstration for In-Building Optical-Wireless Network With Integrated Broadband Services Lightwave Technology, Journal of 27 1920-7

[12] Flatman A 2004 In-premises optical fiber installed base analysis to 2007. In: IEEE 802 . 310 GBE over FDDI-grade fibre Study Group meeting (New York: IEEE)

[13] Das A, Nkansah A, Gomes N J, Garcia I J, Batchelor J C and Wake D 2006 Design of low-cost multimode fiber-fed indoor wireless networks Microwave Theory and Techniques, IEEE Transactions on $\mathbf{5 4} 3426-32$

[14] Morant M, Quinlan T, Llorente R and Walker S 2011 Full standard triple-play bidirectional and full-duplex CWDM transmission in passive optical networks. In: Optical Fiber Communication Conference and Exposition (OFC/NFOEC), 2011 and the National Fiber Optic Engineers Conference, (New York: IEEE) pp 1-3

[15] Perez J, Morant M, Llorente R and Marti J 2009 Joint Distribution of PolarizationMultiplexed UWB and WiMAX Radio in PON Journal of Lightwave Technology 27 1912-9

[16] Saleh A, Rustako A and Roman R 1987 Distributed Antennas for Indoor Radio Communications Communications, IEEE Transactions on 35 1245-51

[17] Ben Ezra Y, Lembrikov B I, Ran M, Leibovich A and Borohovich E 2008 Experimental and theoretical investigation of the multiband OFDM ultra-wideband radio over multimode fiber transmission. In: Ultra-Wideband, 2008. ICUWB 2008. IEEE International Conference on, (New York: IEEE) pp 55-8 
[18] Casini A and Faccin P 2003 Wavelength division multiplation technologies for UMTS radio coverage extension by using the radio over fibre technique. In: Microwave Photonics, 2003. MWP 2003 Proceedings. International Topical Meeting on, (New York: IEEE) pp 123-8

[19] Jensen J B, Rodes R, Caballero A, Yu X, Gibbon T B and Monroy I T 20094 Gbps impulse radio (IR) ultra-wideband (UWB) transmission over 100 meters multi mode fiber with 4 meters wireless transmission Optics Express 17 16898-903

[20] Snow C, Lampe L and Schober R 2007 WiMAX Interference to MB-OFDM UWB Systems. In: Communications, Computers and Signal Processing, 2007. PacRim 2007. IEEE Pacific Rim Conference on, (New York: IEEE) pp 329-32

[21] Alemany R, Perez J, Llorente R, Polo V and Marti J 2008 Coexistence of WiMAX 802.16d and MB-OFDM UWB in radio over multi-mode fiber indoor systems. In: Microwave Photonics, 2008; jointly held with the 2008 asia-pacific microwave photonics conference. MWP/APMP 2008. International topical meeting on, (New York: IEEE) pp 74-7

[22] Guo Y X, Viet Hung P, Yee M L, Ong L C and Luo B 2007 Performance Study of MB-OFDM Ultra-Wideband Signals over Multimode Fiber. In: Ultra-Wideband, 2007. ICUWB 2007. IEEE International Conference on, (New York: IEEE) pp 429-31

[23] Lethien C, Loyez C, Vilcot J-P, Clavier L, Bocquet M and Rolland P A 2009 Indoor coverage improvement of MB-OFDM UWB signals with radio over POF system Optics Communications 282 4706-15 